\title{
ЗАСТОСУВАННЯ МЕТОДУ ЛІНІЙНОГО СПРЯЖЕННЯ У ЗМШАНІЙ ЗАДАЧІ ЗГИНУ ТРАНСТРОПНИХ ПЛИТ
}

\section{APPLICATION OF LINEAR CONJUGATION METHOD FOR MIXED FLEXURAL PROBLEM IN TRANSTROPIC PLATES}

Шваб'юк В.І., д.т.н., проф., Ротко С.В., к.т.н., доц., Шваб’юк В.В., к.т.н., доц., Ужегова О.А., к.т.н., доц., Пасічник Р.В., к.т.Н., доц. (Луцький національний технічний університет)

Shvabyuk V.I., Doctor of Engineering, Professor, Rotko S.V., Ph.D. in Engineering, Associate Professor, Shvabyuk V.V., Ph.D. in Engineering, Associate Professor, Uzhehova O.A., Ph.D. in Engineering, Associate Professor, Pasichnyk R.V., Ph.D. in Engineering, Associate Professor (Lutsk National Technical University, Lutsk)

Розглядається задача згину трансверсально ізотропної шарнірно обпертої плити розподіленим по лінії дії моментним навантаженням. На основі методу лінійного спряження аналітичних функцій отримано розв'язок задачі, а також вирази для згинальних моментів, поперечних сил і напружень у плиті. Одержані формули враховують вплив деформацій поперечного зсуву та обтиснення.

Formulas and dependences derived in works [1,2] for plates made of orthotropic material were used for solving the flexural problem of a hinged edge supported semi-infinite plate. These formulas are written through the analytic functions of a complex variable. The formulas are given for the case of a transversally isotropic material, when certain parameters must satisfy equality.

A new refined model formulas representation of transtropic plates bending in the complex form is proposed in the article. This form is convenient for application of the linear conjugation of analytic functions method by M.I. Mushelishvili. The linear conjugation method of analytic functions is convenient for solving problems with mixed boundary conditions at the edges, or by a piecewise distributed moment load along the support line in particular.

At the same time, it is assumed that torque and displacement of the median plate surface on this boundary are equal to zero. It means that in this case we assume that condition of zero equality at the boundary of 
generalized rotation angle (instead of the torque) does not significantly affect the general solution of the problem.

The solution method for plane problem of the elasticity theory and bending problems of Kirchhoff's thin plates is elaborated in detail in the works of M.I. Muskhelishvili and I.O. Prusov. In this paper, the equations obtained by authors differ from the well-known representations of S. Timoshenko's theories by members, which consider the transverse compression and nonlinearity of the normal stresses distribution in thickness of the plate. This non-linearity is provided by dependencies consideration of transverse forces. Ratios of displacement modules are the multipliers in these dependencies, which can be quite significant.

In the considered case for transtropic material, the complex potentials of M.I. Muskhelishvili can be expressed through one complex variable $z=x+i y$. Formulas for displacements, generalized rotation angles, bending moments and transverse forces in a transtropic plate could also be expressed in a compact form through the complex potentials.

Ключові слова: транстропна плита, уточнена модель згину, поперечний зсув та обтиснення, метод лінійного спряження.

Keywords: transtropic plate, refined bending model, transverse shear and pressurizing, linear conjugation method.

Вступ. Для розв'язання задачі згину шарнірно обпертої на краю напівнескінченної плити використано формули та залежності, виведені у роботах [1,2] для плит із ортотропного матеріалу i записані через аналітичні функції комплексної змінної. Формули наведені для випадку трансверсально-ізотропного матеріалу, коли певні параметри мають задовольняти рівності $\alpha=\lambda=1$. У статті запропоновано нове подання формул уточненої моделі згину транстропних пластин у комплексній формі, зручній для застосування методу лінійного спряження аналітичних функцій M.I. Мусхелішвілі [3]. Метод лінійного спряження аналітичних функцій зручно використовувати для розв'язування задач із змішаними граничними умовами на краях [4,5] або, зокрема, розривним згинальним навантаженням уздовж лінії обпирання. 


\section{Викладення основного матеріалу}

Методика розв'язування таких задач для плоскої задачі теорії пружності та задач згину тонких пластинок Кірхгофа детально розроблена у працях М.I. Мусхелішвілі [3] та I.О. Прусова [4,5]. У даній роботі отримані авторами рівняння відрізняються від уже відомих представлень теорій типу С.Тимошенка членами, які враховують поперечне обтиснення, а також нелінійність розподілу нормальних напружень по товщині пластини.

У розглядуваному випадку для транстропного матеріалу комплексні потенціали М.I. Мусхелішвілі можна виразити через одну комплексну змінну $z=x+i y$, а формули для переміщень, узагальнених кутів повороту, згинальних моментів і поперечних сил у транстропній плиті можна подати в компактному вигляді $[1,2]$ :

$$
\begin{gathered}
M_{y}+M_{x}=-2 D(1+v)\left[\Phi(z)+\overline{\Phi(z)}[\Phi(z)+\overline{\Phi(z)}]+M_{y}^{*}+M_{x}^{*}\right. \\
M_{y}-M_{x}+2 i H_{x y}=4 D_{6}\left[\Psi(z)+\bar{z} \Phi^{\prime}(z)+0.4 i \bar{R}_{2}\right]+M_{y}^{*}-M_{x}^{*}+2 i H_{x y}^{*} \\
M_{x}-v M_{y}-i(1+v) H_{x y}= \\
=-D\left(1-v^{2}\right)\left[\Phi(z)+\overline{\Phi(z)}+\Psi(z)+\bar{z} \Phi^{\prime}(z)+\frac{2}{5} i\left(\bar{R}_{2}\right)\right]+M^{*} \\
Q_{x}-i Q_{y}=-4 D \Phi(z)-i K^{\prime} \bar{R}_{1}+Q_{x}^{*}-i Q_{y}^{*} \\
\gamma_{x}+i \gamma_{y}=-[\varphi(z)+z \overline{\Phi(z)}+\overline{\psi(z)}]+0.8 i R_{1}+\gamma_{x}^{*}+i \gamma_{y}^{*} \\
\frac{\partial w}{\partial x}+i \frac{\partial w}{\partial y}=-\left(\gamma_{x}+i \gamma_{y}\right)-4 \varepsilon_{1} \overline{\Phi^{\prime}(z)}+\frac{4}{5} i R_{1},
\end{gathered}
$$

де $M^{*}=M_{x}^{*}-v M_{y}^{*}-i(1+v) H_{x y}^{*} ; R_{1}=\Omega_{x}^{\prime}+i \Omega_{y}^{\prime}$;

$$
R_{2}=\left(\frac{\partial}{\partial x}+i \frac{\partial}{\partial y}\right)^{2} \Omega ; \quad \varepsilon_{1}=\left(8 \frac{G}{G^{\prime}}-3 v^{\prime \prime}\right) \frac{h^{2}}{10(1-v)}
$$

Величини $M_{x}^{*}, M_{y}^{*}, H_{x y}^{*}, Q_{x}^{*}, Q_{y}^{*}, w^{*}, \gamma_{x}^{*}, \gamma_{y}^{*}$ визначаються за рахунок часткових розв'язків системи рівнянь [6]:

$$
D \Delta^{2} w=q_{2}-\varepsilon_{1} \Delta q_{2}-\varepsilon_{2} \Delta^{2} q_{2},
$$




$$
\Delta \Omega-k_{0}^{2} \Omega=0 ; K^{\prime} \Delta \tilde{w}_{\tau}=-q_{2},
$$

де $\varepsilon_{2}=\left(1-0,4 v^{\prime} A^{\prime}(3-2 v)\right) \frac{h^{4}}{20} \frac{\tilde{E}}{E^{\prime}}, \tilde{E}=\frac{E}{\left(1-v^{2}\right)} ; k_{0}^{2}=\frac{5}{2} \frac{G^{\prime}}{G} h^{-2}$.

Для випадку трансверсально-ізотропного матеріалу ці величини знаходяться із співвідношень:

$$
\begin{aligned}
& M_{x}^{*}+M_{y}^{*}=-4 D(1+v) \frac{\partial w^{*}}{\partial z \partial \bar{z}}+\frac{4}{5} A^{\prime} q_{2} h^{2} \\
& M_{y}^{*}-M_{x}^{*}+2 i H_{x y}^{*}=4 D(1-v) \frac{\partial^{2} w^{*}}{\partial z^{2}} ; Q_{x}^{*}+i Q_{y}^{*}=-8 D \frac{\partial^{3} w^{*}}{\partial z^{2} \partial \bar{z}} \\
& \frac{\partial \tilde{w}^{*}}{\partial x}+i \frac{\partial \tilde{w}^{*}}{\partial y}=2 \frac{\partial \tilde{w}^{*}}{\partial \bar{z}} ; \gamma_{x}^{*}+i \gamma_{y}^{*}=-2 \frac{\partial \tilde{w}^{*}}{\partial \bar{z}} .
\end{aligned}
$$

Формулами (3) зручно користуватись, коли пластинка згинається зусиллями або моментами, розподіленими по краю, а навантаження на поверхнях $\gamma= \pm h$ відсутнє: $q_{2}(z, \bar{z})=0$. Аналіз системи рівнянь (2) для трансверсально-ізотопних пластин показує, що їх згин описується однією бігармонійною функцією $w(x, y)$, однією гармонійною $w_{\tau}(x, y)$ і функцією $\Omega(x, y)$, що задовольняє рівнянню Гельмгольца. Надалі будемо припускати, що функція $\Omega \epsilon$ однозначною в будь-якій точці однозв'язної, чи багатозв'язної області, яку займає пластина і прямує до нуля при $|z| \rightarrow \infty$. Тому згадану функцію можна записати у вигляді [4]:

$$
\Omega(x, y)=\int_{0}^{\infty} C(\tau) e^{\tau \omega y} \sin \tau x d \tau,
$$

де $\omega=\sqrt{1+k_{0}^{2} \tau^{-2}}, C(\tau)$ - дійсна функція параметра $\tau$.

Одночасно, вирази і інтеграли, котрі мають функції $\Omega(x, y)$, чи $R_{1}(x, y)$, можна покласти рівними нулю при будь-якому замкнутому контурі. Тим самим вважається, що головний вектор i головний момент напруженого стану, який описується цією 
функцією на боковій поверхні, і вирізаного 3 пластини циліндра, дорівнює нулю.

У випадку, коли рівняння (2) стають однорідними $\left(q_{2}(z, \bar{z})=0\right)$, у формулах (1) необхідно покласти

$$
M_{x}^{*}=M_{y}^{*}=H_{x y}^{*}=Q_{x}^{*}=Q_{y}^{*}=w^{*}=\gamma_{x}^{*}=\gamma_{y}^{*}=0 .
$$

У результаті, отримаємо наступні системи рівнянь, які можна використати при постановці граничних задач для транстропної плити:

$$
\begin{gathered}
\Phi(z)+\Phi(\bar{z})+(z-\bar{z}) \overline{\Phi^{\prime}(z)}=-\eta_{1} f_{1}+0,4 i R_{2}-\Psi_{0}(\bar{z}) \\
\kappa \Phi(z)+\Phi(\bar{z})-(z-\bar{z}) \overline{\Phi^{\prime}(z)}=-\eta_{2} f_{2}-\frac{2}{5} i\left(R_{2}+k_{0}^{2} \Omega\right)+\Psi_{0}(\bar{z}) \\
2 i \cdot D[\Phi(z)-\overline{\Phi(z)}]=K^{\prime} \Omega-P_{y}+C_{0} \\
\Phi(z)-\Phi(\bar{z})+(z-\bar{z}) \overline{\Phi^{\prime}(z)}=g(x)+0.8 i\left(R_{1}\right)_{x}^{\prime} \\
4 \varepsilon_{1} \overline{\Phi^{\prime}(z)}=\frac{4}{5} i R_{1}-\left[\left(\gamma_{x}+\frac{\partial w}{\partial x}\right)+i\left(\gamma_{y}+\frac{\partial w}{\partial y}\right)\right]
\end{gathered}
$$

де $f_{1}(x)=M_{x}-M_{y}+i(1+v) H_{x y}$;

$$
\begin{gathered}
f_{2}(x)=M_{y}-i\left(H_{x y}+P_{y}+C_{0}\right) ; \kappa=(3+v)(1-v)^{-1} ; \\
g(x)=-\frac{\partial}{\partial x}\left(\gamma_{x}+i \gamma_{y}\right)-\Psi_{0}(\bar{z}) ; P_{y}=\int_{x_{0}}^{x} Q_{y} d x ; \\
D_{6}=\frac{2}{3} G h^{3}, \eta_{1}=1 /\left(D\left(1-v^{2}\right)\right), \eta_{2}=0,5 D_{6} ; \\
\kappa_{0}^{*}=\left(1+v_{12}\right)(1-v)^{-1} ; K^{\prime}=\frac{4}{3} G^{\prime} h, D=\frac{2}{3} \tilde{E} h^{3}, D_{12}=v D ; \\
C_{0}-\text { довільна дійсна стала, яка дорівнює } P_{y} \text { у точці } x_{0} .
\end{gathered}
$$


Необхідно зауважити, що комплексні потенціали М.І. Мусхелішвілі у формулах і рівняннях (1), (4) : $\Phi(z)=\frac{d}{d z} \varphi(z)$ і $\Psi(z)=\frac{d}{d z} \psi(z)$, де $\varphi(z), \psi(z)=\frac{d}{d z} \chi(z)$, використовуються для представлення переміщення $w$ у вигляді формули Гурса $[3,4]$ :

$$
w=\operatorname{Re}[\bar{z} \cdot \varphi(z)+\chi(z)]+w * .
$$

Надалі будемо вважати, що на нескінченності пластина може бути навантажена відомими скінченими моментами $M_{x}^{\infty}, M_{y}^{\infty}$, $M_{x y}^{\infty}$. Тому при $|z| \rightarrow \infty$ функції $\Phi(z)$ і $\Psi(z)$ мають вид

$$
\Phi(z)=\Gamma+a_{\infty} / z+O\left(1 / z^{2}\right) ; \Psi(z)=\Gamma^{\prime}+\bar{a}_{\infty} / z+O\left(1 / z^{2}\right)
$$

де $\quad \Gamma=-\frac{M_{x}^{\infty}+M_{y}^{\infty}}{4 D_{1}(1+v)} ; \quad \Gamma^{\prime}=\frac{M_{y}^{\infty}-M_{x}^{\infty}+2 i H_{x y}^{\infty}}{4 D_{6}} ; a_{\infty}=\frac{m^{\prime}}{8 i \pi D}$. $m^{\prime}=m_{x}^{\infty}+i m_{y}^{\infty}-$ головний момент відносно початку координат всього зовнішнього навантаження, прикладеного до транстропної пластини.

На прикладі згину півнескінченної шарнірно опертої плити кусково-розподіленим моментним навантаженням необхідно визначити напружено-деформований стан i вплив на нього деформацій поперечного зсуву та обтиснення, особливо в околі точок розриву неперервності навантаження, прикладеного до краю плити. Вважається, що серединна поверхня недеформованої плити співпадає із координатною півплощиною $y<0$ (обл. $S^{-}$), а межа $L$ із віссю $y=0$. Плита згинається сталим розподіленим моментним навантаженням $m$, що діє відрізку $(-a, a)$ межі $L$. Крайові умови на межі $L$ записуються наступним чином:

$$
\begin{aligned}
& M_{y}=m=\text { const на } L(-a \leq x \leq a), \\
& M_{y}=0 \quad \text { на } L^{\prime \prime}=L-L^{\prime} ; \\
& H_{x y}=0,\left.W\right|_{\gamma=\gamma_{0}}=0 \text { на } L .
\end{aligned}
$$


На основі виразів (1), (4) крайові умови зводяться до вигляду:

$$
\begin{gathered}
\kappa^{*} \Phi^{+}(x)+\Phi^{-}(x)+\left(1+\kappa^{*}\right) \overline{\Phi^{-}(x)}=-2 \int_{0}^{\infty} F(\tau) \cos \tau x d \tau+ \\
\quad+0,4 \kappa^{*} \int_{0}^{\infty} \tau^{2} C(\tau)\left[2 \omega \cos \tau x+i\left(1+\omega^{2}\right) \sin \tau x\right] d \tau, \\
\operatorname{Re}\left[\Phi^{-}(x)-\Phi^{+}(x)-\left(\varepsilon_{1}-2 A^{\prime} z_{0}^{2}\right) \Phi^{-\prime \prime}(x)\right]=0, \\
\text { де } A^{\prime}=\frac{v^{\prime \prime}}{1-v} ; F(\tau)=m[D \pi(1+v) \tau]^{-1} \sin \tau a .
\end{gathered}
$$

Розв'язок крайової задачі (8) для $\Phi(z)$ можна взяти у вигляді [1]:

$$
\Phi(z)=\int_{0}^{\infty} A(\tau) e^{-i \tau z} d \tau \text { при } z \in D^{-}, \Phi(z)=\int_{0}^{\infty} A(\tau) e^{i \tau z} d \tau \text { при } z \in D^{+},
$$

тут $A(\tau), B(\tau)$ і $C(\tau)$ - невідомі функції, які визначаються із крайових умов (8):

$$
\begin{gathered}
A(\tau)=-\frac{2\left(1+\omega^{2}\right) F(\tau)}{\kappa^{*}(\omega-1)^{2}\left[2+\tau^{2}\left(\varepsilon_{1}-2 A^{\prime} z_{0}^{2}\right)\right]+2\left(1+\omega^{2}\right)} \\
B(\tau)=-\frac{5\left[2+\tau^{2}\left(\varepsilon_{1}-2 A^{\prime} z_{0}^{2}\right)\right] F(\tau)}{\kappa^{*}(\omega-1)^{2}\left[2+\tau^{2}\left(\varepsilon_{1}-2 A^{\prime} z_{0}^{2}\right)\right]+2\left(1+\omega^{2}\right)} \\
C(\tau)=-\frac{2\left(1+\omega^{2}\right)\left[1+\tau^{2}\left(\varepsilon_{1}-2 A^{\prime} z_{0}^{2}\right)\right] F(\tau)}{\kappa^{*}(\omega-1)^{2}\left[2+\tau^{2}\left(\varepsilon_{1}-2 A^{\prime} z_{0}^{2}\right)\right]+2\left(1+\omega^{2}\right)}
\end{gathered}
$$

Необхідно зауважити, що рівняння (1), (4) по формі співпадають 3 відповідними рівняннями І.О. Прусова [4] для транстропного матеріалу і відрізняються тільки коефіцієнтами, що враховують вплив поперечного обтиснення. У класичній постановці подібні дослідження наведені у роботі А.І. Каландії [7].

Вирази для згинального момента $M_{x}^{-}$та поперечної сили $Q_{y}^{-}$ знаходимо із відповідних формул $[1,6]$ у вигляді: 
Tyт

$$
\begin{gathered}
M_{x}^{-}=4 m \int_{0}^{\infty}(\pi t)^{-1} f_{1}(t) \sin \eta t \cos \xi t d t-\varepsilon m \\
Q_{y}^{-}=-\frac{4 m}{\pi(1+v) h} \int_{0}^{\infty} t^{-2} f_{2}(t) \sin \eta t \cos \xi t d t \\
f_{1}(t)=\left[1+\kappa^{*}\left(1+m_{2} t^{2}\right)\left(1-\frac{2 t \sqrt{t^{2}+2,5 G^{\prime} / G}}{2 t^{2}+2,5 G^{\prime} / G}\right)\right]^{-1} ;
\end{gathered}
$$

$$
\begin{aligned}
& f_{2}(t)=\frac{2 t^{4}+1,25(1-v)\left(1+m_{2} t^{2}\right)\left(1+2,5 t^{2}\right) G^{\prime} / G}{2 t^{2}+2,5 G^{\prime} / G+\left(1+m_{2} t^{2}\right)\left(\sqrt{t^{2}+2,5 G^{\prime} / G}-t\right)^{2}}, \\
& m_{2}=\frac{2}{3(1-v)}\left(2 \frac{G}{G^{\prime}}-v^{\prime \prime}\right), \eta=\frac{a}{h}, \xi=\frac{x}{h}, \varepsilon-\text { кусково-стала }
\end{aligned}
$$

величина, яка рівна одиниці на $L^{\prime}$ і нулю на $L^{\prime \prime}$.

Визначивши згинальні моменти та поперечні сили, напруження $\sigma_{x}, \sigma_{y}$ для трансверсально-ізотропної плити можна представити у вигляді [1]:

$$
\begin{array}{r}
\sigma_{x}=\frac{3 M_{x}}{2 h^{3}} \gamma+\frac{3}{2} \cdot \frac{G}{G^{\prime}} \cdot\left(\frac{\partial Q_{x}}{\partial x}+v \frac{\partial Q_{y}}{\partial y}\right) \cdot f(\gamma), \\
\sigma_{y}=\frac{3 M_{y}}{2 h^{3}} \gamma+\frac{3}{2} \cdot \frac{G}{G^{\prime}} \cdot\left(\frac{\partial Q_{y}}{\partial y}+v \frac{\partial Q_{x}}{\partial x}\right) \cdot f(\gamma), \\
\text { де } f(\gamma)=\frac{\gamma}{5 h}\left(1-\frac{5}{3} \cdot \frac{\gamma^{2}}{h^{2}}\right)(1-v)^{-1} .
\end{array}
$$

Поклавши $\gamma= \pm h$, будемо мати значення напружень $\sigma_{x}, \sigma_{y}$ на зовнішніх поверхнях плити:

$$
\begin{aligned}
& \sigma_{x}= \pm \frac{3 M_{x}}{2 h^{2}} \mp \frac{G / G^{\prime}}{5(1-v)}\left(\frac{\partial Q_{x}}{\partial x}+v \frac{\partial Q_{y}}{\partial y}\right), \\
& \sigma_{y}= \pm \frac{3 M_{y}}{2 h^{2}} \mp \frac{G / G^{\prime}}{5(1-v)} \cdot\left(\frac{\partial Q_{y}}{\partial y}+v \frac{\partial Q_{x}}{\partial x}\right) .
\end{aligned}
$$


Вираз в дужках (12), (13) записується через функції $\Phi(\tilde{z})$ i $\Omega(x, y)$ наступним чином:

$$
\frac{\partial Q_{x}}{\partial x}+v \frac{\partial Q_{y}}{\partial y}=-2(1-v) D\left[\Phi^{\prime \prime}(z)+\overline{\Phi^{\prime \prime}(z)}\right]-(1-v) \cdot K^{\prime} \cdot \frac{\partial^{2} \Omega}{\partial x \partial y} .
$$

У формулах (12), (13) тільки перші члени, відповідають теоріям типу С.П.Тимошенка [4]. Другі члени є уточненнями формул для $\sigma_{x}, \sigma_{y}$ за рахунок нелінійності відносно вертикальної координати $\gamma$ і при великих відношеннях $G / G^{\prime}$ можуть бути досить значними.

Висновок. На основі методу лінійного спряження аналітичних функцій отримано розв'язок задачі згину трансверсально ізотропної півнескінченної шарнірно опертої плити кусково-розподіленим моментним навантаженням, що діє уздовж відрізка границі плити. Отримано вирази для згинальних моментів, поперечних сил i напружень у плиті. Одержані формули враховують вплив деформацій поперечного зсуву та обтиснення, а також поведінку цих величин в околі точок розриву неперервності навантаження.

\section{Список використаних джерел}

1. Шваб'юк B.I. Комплексне подання уточнених рівнянь згину ортотропних пластин 3 тріщинами / В.І. Шваб’юк // Машинознавство. 1999.- №4.- С. 51-55. 2. Швабюк В.І. Розрахунок некласичної моделі згину ортотропних плит методом лінійного спряження / Швабюк В.I., Ротко С.В., Ужегова О.А., Гуда О.В. // Зб. наукових праць „Сучасні технології та методи розрахунків у будівництві”. - Луцьк: Видавництво ЛНТУ. - 2015. - Вип. 3. - С. 220-227. 3. Мусхелишвили Н.И. Некоторые основные задачи математической теории упругости / Н.И. Мусхелишвили. - М.: Наука, 1966. - 707c. 4. Прусов И.А. Метод сопряжения в теории плит / И.А. Прусов. - Минск: Издательство Беларусского государственного университета, 1975. - 256c. 5. Прусов И.А., Швабюк В.И. Изгиб полубесконечной плиты, частино защемленной, частично шарнирно опертой. /Сб. Сопротивление материалов и теория сооружений. К., Изд-во «Будівельник», 1970. В. 12. С. 50-54. 6. Швабюк В.И. Учет эффекта сжимаемости нормали в контактных задачах для трансверсальноизотропных плит / В.И. Швабюк // Прикл. механика. - 1980. - Т. 16. - №9. - С. 71-77. 7. Каландия А.И. Об одной смешанной задаче изгиба упругой пластинки./ А.И. Каландия. - М. - ПММ .1952. Т. 16. В. 3. С. 43-51. 